\title{
Supporting Information for \\ Chemical composition, crystal morphology, and key gene expression of the cuticular waxes of goji (Lycium barbarum L.) berries $^{1}$
}

Peng Wang ${ }^{\mathrm{a}}$, Junjie Wang ${ }^{\mathrm{a}, *}$, Huaiyu Zhang ${ }^{\mathrm{a}}$, Cong Wang ${ }^{\mathrm{a}}$, Lunaike Zhao ${ }^{\mathrm{a}}$, Ting Huang ${ }^{\mathrm{b}}$, Ken Qing $^{\mathrm{b}}$

${ }^{a}$ Key Laboratory of Storage and Processing of Plant Agro-Products, College of Biological Science and Engineering, North Minzu University, Yinchuan 750021, China.

${ }^{\mathrm{b}}$ National Wolfberry Engineering Research Center, Ningxia Academy of Agricultural and Forestry Sciences, Yinchuan 750002, China.

Corresponding author. College of Biological Science and Engineering, North Minzu University, Yinchuan 750021, China.

* E-mail address: smkxwjj@163.com (J. Wang) 


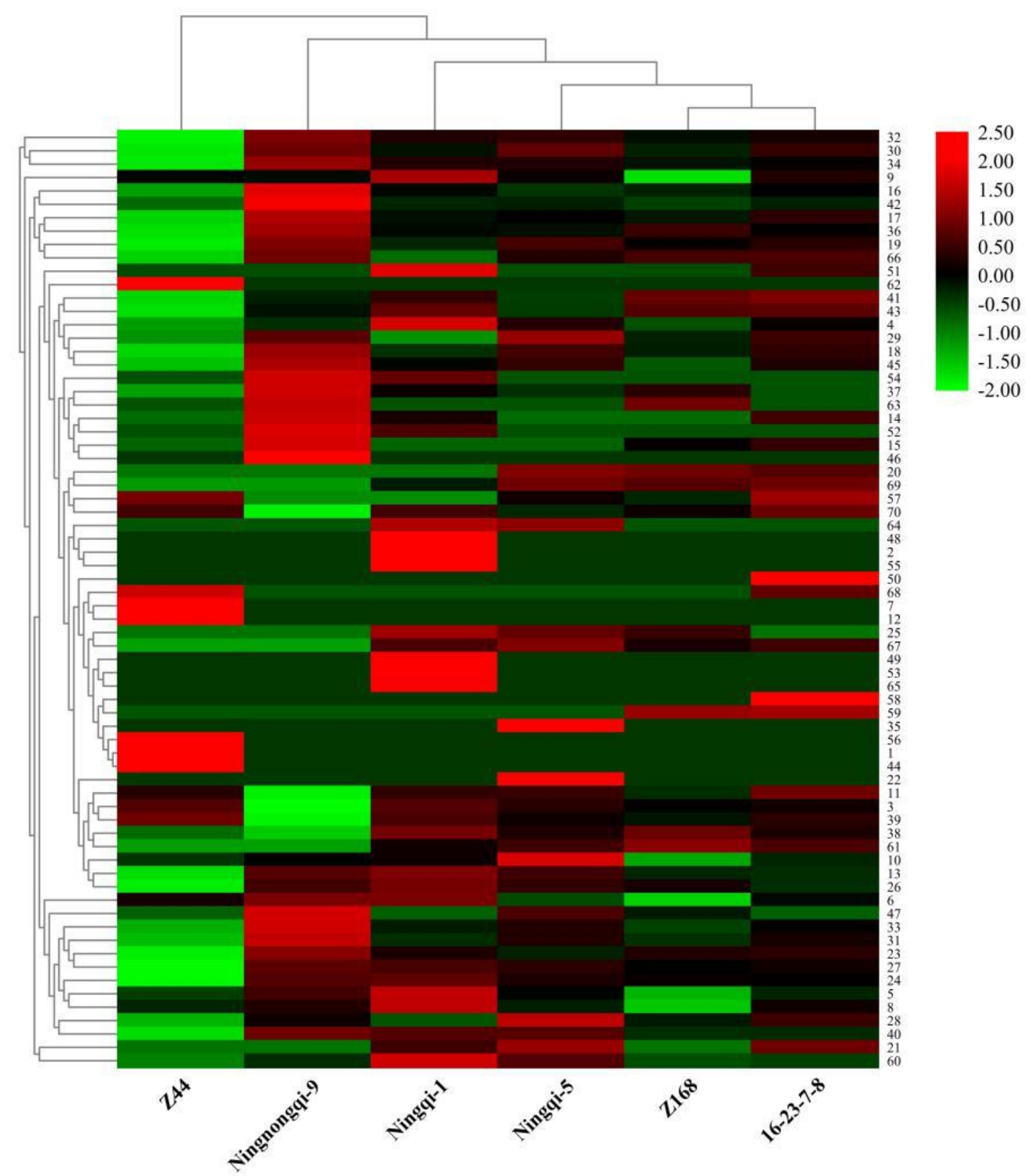

Figure S1. Heatmap and clustering of 70 wax components in 6 goji berry cultivars. Colors indicate chemical composition levels. The numbers of each row represent the wax component numbers corresponding to the accession numbers in Supplementary Table S2. Each column represents a goji berry cultivar. 

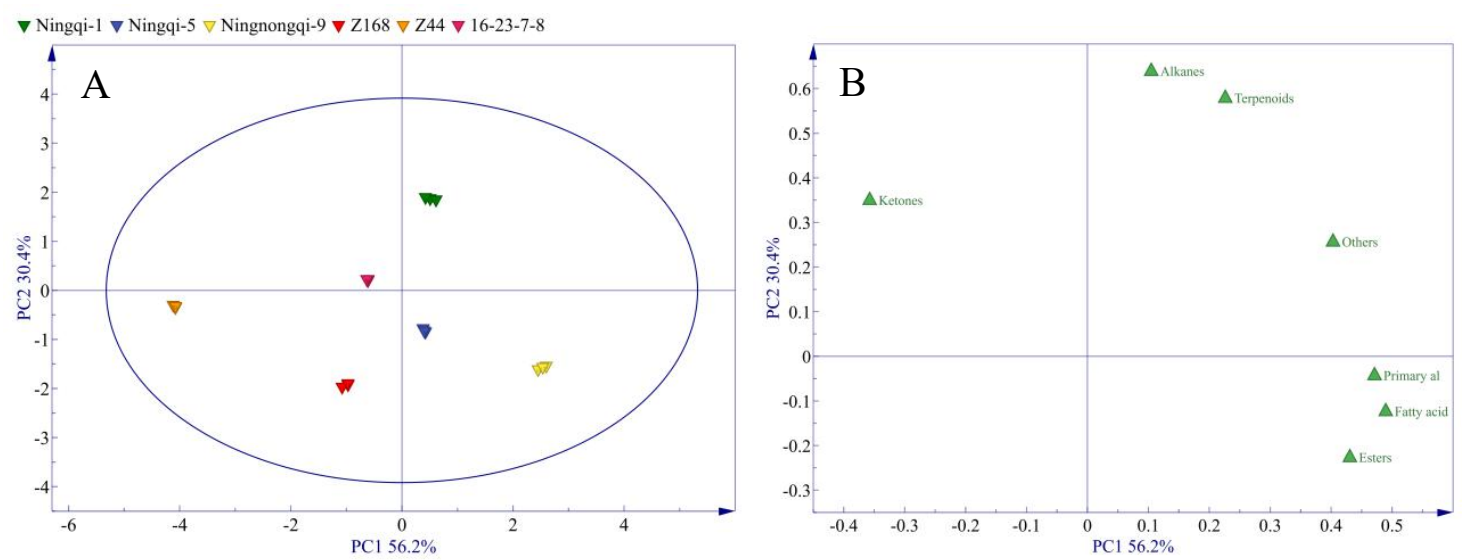

Figure S2. PCA of seven main classes of wax compounds in six goji berry cultivars. PCA score plots (A) and loading plots (B) of the chemical compositions of the cuticular wax in six goji berry cultivars. The numbers in (B) correspond to the sample numbers given in Table S3. Percentages in parentheses are the variance of each component. 

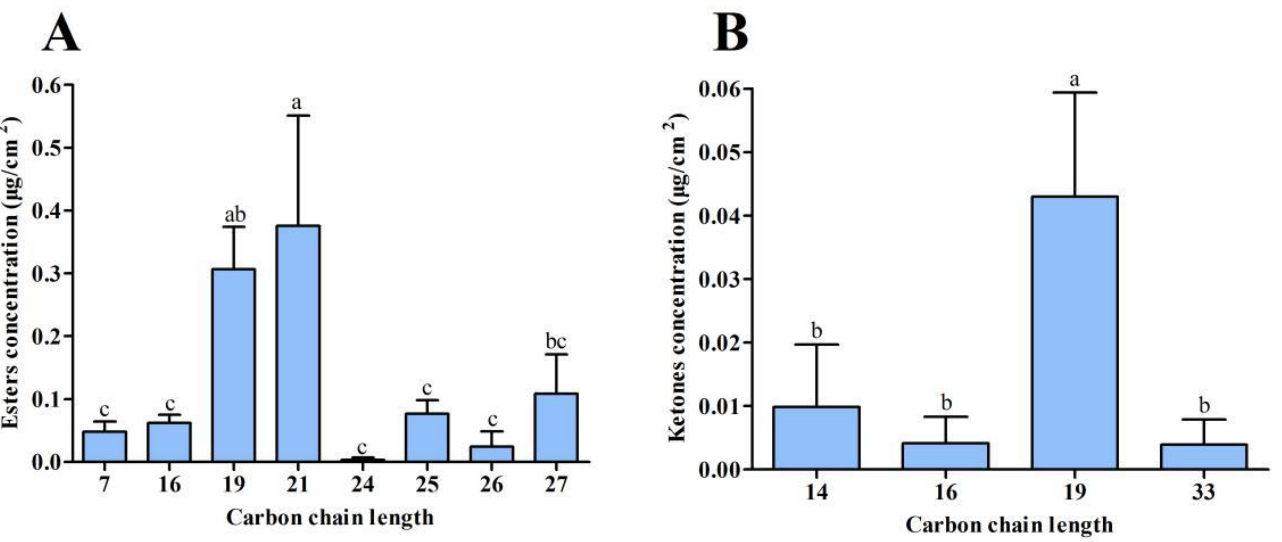

Figure S3. Distribution and concentration of the main VLC aliphatic compounds detected in the epicuticular wax of eight goji berry cultivars. (A) Esters; (B) ketones. Each column represents the average of six varieties. Statistical significance was determined using Duncan' s multiple range tests. Each value is the mean SD of eighteen biological repeats. Different letters above each bar indicate significant differences at the 5\% level. 

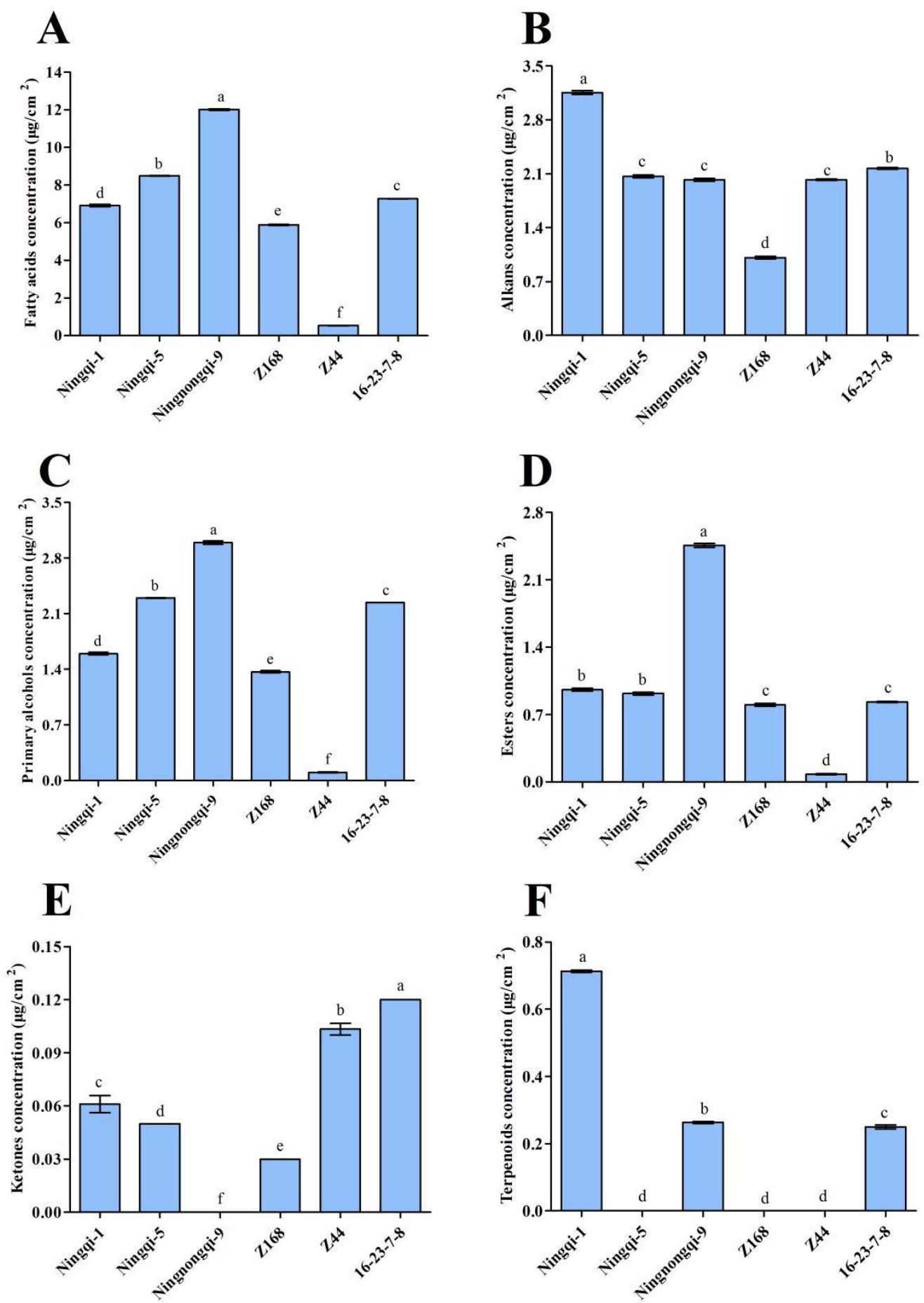

Figure S4. Amounts of the six main classes of wax compounds in the fruit of eight goji berry cultivars. (A) Fatty acids; (B) alkanes; (C) primary alcohols; (D) esters; (E) ketones; (F) terpenoids. Statistical significance was determined using Duncan's multiple range tests. Each value is the mean SD of three biological repeats. Different letters above each bar indicate significant differences at the $5 \%$ level. 

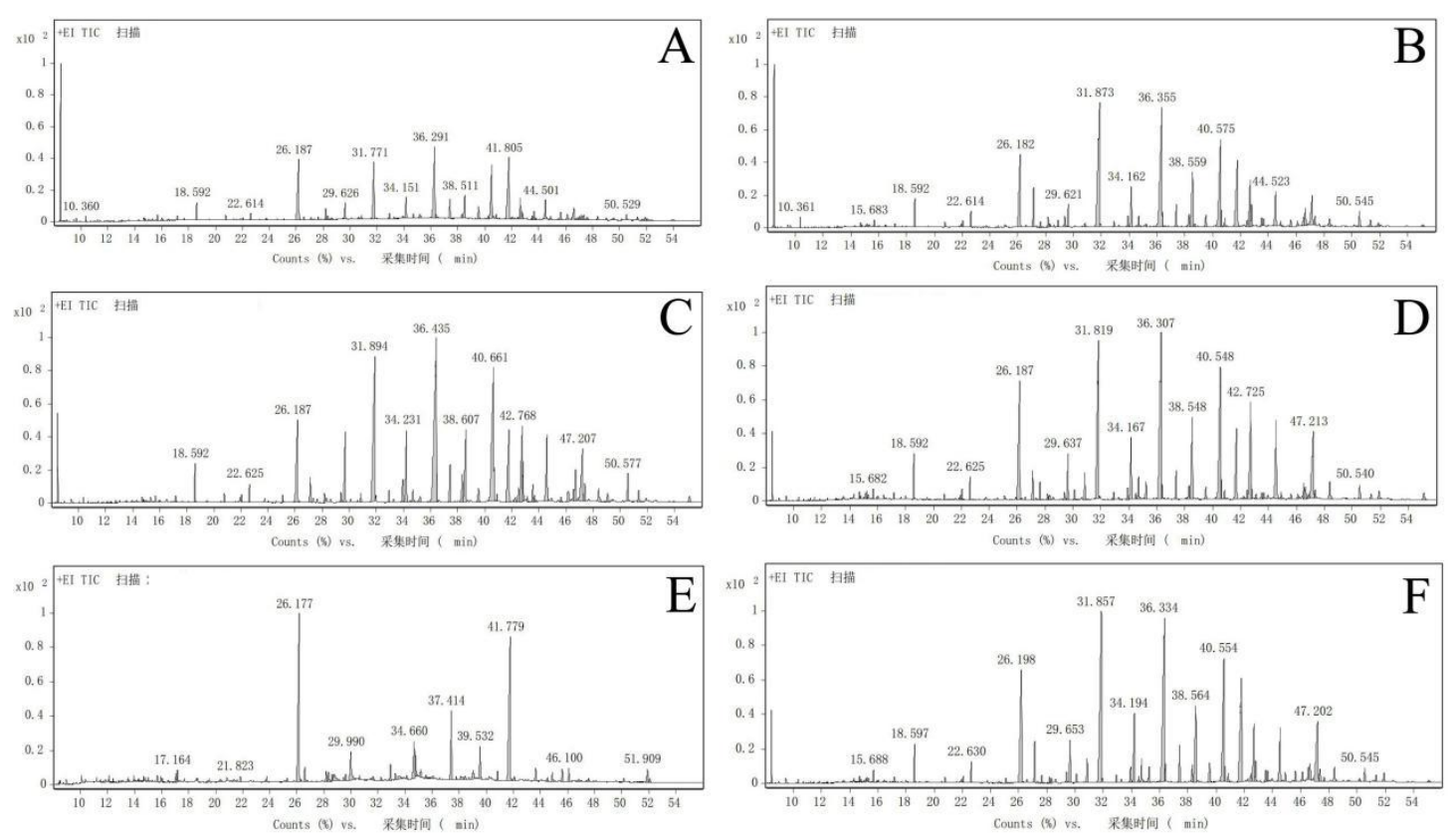

Figure S5. A chromatogram of the chemical composition of the epicuticular wax from six goji berries, as analyzed using gas chromatography. (A) 'Ningqi-1'; (B) 'Ningqi-5'; (C) ) 'Ningnongqi-9'; (D) 'Z168'; (E) 'Z44' and (F) '16-23-7-8'. Each sample was measured as three biological repeats. 
Table S1. Primer sequences used for quantitative real-time RT-PCR.

\begin{tabular}{lllc}
\hline Gene & Forward $\left(5^{\prime}-3^{\prime}\right)$ & Reverse $\left(5^{\prime}\right.$-3') & Product(bp) \\
\hline Actin & CTCAGCACCTTCCAGCAGAT & TAACACTGCAACCGCATTTC & 162 \\
EF- $1 \alpha$ & GAAGGGTGTCCCTCAGATCA & CCGTCCATGTCGTCTCTTTT & 180 \\
CER1 & GCTCCATGTGCCAGTCACTTAGG & AACATCACCACGCTAGGACCATTG & 214 \\
CER6 & TGCCACCTTCAACTCATTACATCC & CAACTTGCACTTGTTCACCACCAT & 206 \\
LACS1 & CGCAAGAATTGTTCCACCTCACTG & CTTCAGGAGGAGCATGTTCCACTT & 195 \\
MAH1 & ATGATAGCAGGGCGTGATACAACT & TGGAATGGCACAGGTGGATATAGC & 227 \\
LTP4 & GCCAGGCGGCTTGTAACTGTATT & CCGTGGAGCAGTCAATGGAAGG & 138 \\
ABC11 & CTTGCGTGGTATAAGTGGAGGAGA & TCGGCTGGTGAATTGAGGCTATC & 191 \\
MYB 96 & GCAGCCATAGCTTCATACCTTCCA & AGCCTCCTTTCCCATTGTCCTTT & 173 \\
$W I N 1$ & TGCCATCACCATCTCTCACTTGTT & TGAAGTTCCACTGTCATCACCCAT & 118 \\
\hline
\end{tabular}




\begin{tabular}{|c|c|c|c|c|c|c|c|c|c|}
\hline \multirow{2}{*}{ Codes } & \multirow{2}{*}{ Compounds } & \multirow{2}{*}{$\mathrm{RI}^{*}$} & \multirow{2}{*}{$\mathrm{RI}^{* * *}$} & \multicolumn{6}{|c|}{ Cultivars } \\
\hline & & & & Ningqi-1 & Ningqi-5 & Ningnongqi-9 & Z168 & Z44 & $16-23-7-8$ \\
\hline 1 & Heneicosane & 2109.00 & 2205.72 & ND & ND & ND & ND & $0.02 \pm 0.00$ & ND \\
\hline 2 & Hexadecane, 8-hexyl- & 2064.00 & 2121.49 & $0.06 \pm 0.00$ & ND & ND & ND & $\mathrm{ND}$ & ND \\
\hline 3 & Pentacosane & 2606.00 & 2516.34 & $0.04 \pm 0.00$ & $0.04 \pm 0.00$ & ND & $0.03 \pm 0.00$ & $0.04 \pm 0.00$ & $0.03 \pm 0.00$ \\
\hline 4 & Hexacosane & 2800.00 & 2717.12 & $0.16 \pm 0.00$ & $0.08 \pm 0.01$ & $0.04 \pm 0.01$ & $0.03 \pm 0.00$ & $\mathrm{ND}$ & $0.07 \pm 0.00$ \\
\hline 5 & Octacosane & 2804.00 & 2717.63 & $0.20 \pm 0.00$ & $0.13 \pm 0.00$ & $0.16 \pm 0.00$ & $0.08 \pm 0.00$ & $0.11 \pm 0.01$ & $0.12 \pm 0.00$ \\
\hline 6 & Nonacosane & 2904.00 & 2920.15 & $0.37 \pm 0.01$ & $0.24 \pm 0.00$ & $0.37 \pm 0.01$ & $0.15 \pm 0.00$ & $0.30 \pm 0.01$ & $0.28 \pm 0.00$ \\
\hline 7 & Octacosane, 2-methyl- & 3161.00 & 3192.42 & $\mathrm{ND}$ & ND & $\mathrm{ND}$ & $\mathrm{ND}$ & $0.06 \pm 0.00$ & ND \\
\hline 8 & Triacontane & 3003.00 & 3016.24 & $0.23 \pm 0.02$ & $0.14 \pm 0.01$ & $0.17 \pm 0.00$ & $0.08 \pm 0.01$ & $0.14 \pm 0.01$ & $0.16 \pm 0.01$ \\
\hline 9 & Hentriacontane & 3103.00 & 3119.37 & $1.98 \pm 0.02$ & $1.31 \pm 0.02$ & $1.22 \pm 0.02$ & $0.56 \pm 0.02$ & $1.24 \pm 0.01$ & $1.39 \pm 0.01$ \\
\hline 10 & Triacontane, 2-methyl - & 3060.70 & 3075.95 & $0.06 \pm 0.00$ & $0.07 \pm 0.00$ & $0.06 \pm 0.00$ & $0.05 \pm 0.00$ & $0.05 \pm 0.00$ & $0.06 \pm 0.00$ \\
\hline 11 & Tritriacontane & 3301.00 & 3259.44 & $0.05 \pm 0.00$ & $0.05 \pm 0.00$ & $\mathrm{ND}$ & $0.03 \pm 0.00$ & $0.05 \pm 0.00$ & $0.06 \pm 0.00$ \\
\hline 12 & Hexadecanol & 1875.00 & 1936.44 & ND & $\mathrm{ND}$ & $\mathrm{ND}$ & ND & $0.06 \pm 0.00$ & ND \\
\hline 13 & Octadecanol & 2081.00 & 2176.45 & $0.06 \pm 0.01$ & $0.05 \pm 0.00$ & $0.05 \pm 0.00$ & $0.03 \pm 0.00$ & ND & $0.03 \pm 0.00$ \\
\hline 14 & Icosanol & 2292.00 & 2374.04 & $0.03 \pm 0.00$ & ND & $0.06 \pm 0.00$ & ND & ND & $0.03 \pm 0.00$ \\
\hline 15 & Batylalcohol & 2608.00 & 2554.54 & $\mathrm{ND}$ & $\mathrm{ND}$ & $0.12 \pm 0.01$ & $0.04 \pm 0.00$ & $\mathrm{ND}$ & $0.06 \pm 0.00$ \\
\hline 16 & Docosanol & 2501.00 & 2573.35 & $0.28 \pm 0.01$ & $0.20 \pm 0.00$ & $0.83 \pm 0.01$ & $0.24 \pm 0.01$ & $0.02 \pm 0.00$ & $0.30 \pm 0.01$ \\
\hline 17 & Tetracosanol & 2650.00 & 2732.62 & $0.38 \pm 0.01$ & $0.42 \pm 0.01$ & $0.89 \pm 0.01$ & $0.37 \pm 0.01$ & $0.02 \pm 0.00$ & $0.53 \pm 0.01$ \\
\hline
\end{tabular}




\begin{tabular}{|c|c|c|c|c|c|c|c|c|c|}
\hline 18 & Pentacosanol & 2749.00 & 2822.32 & $0.05 \pm 0.00$ & $0.08 \pm 0.00$ & $0.11 \pm 0.00$ & $0.05 \pm 0.01$ & ND & $0.08 \pm 0.00$ \\
\hline 19 & Hexacosanol & 2852.00 & 2951.92 & $0.43 \pm 0.01$ & $0.73 \pm 0.00$ & $0.94 \pm 0.03$ & $0.56 \pm 0.00$ & $\mathrm{ND}$ & $0.65 \pm 0.00$ \\
\hline 20 & Heptacosanol & 3016.00 & 3064.29 & $\mathrm{ND}$ & $0.09 \pm 0.00$ & $\mathrm{ND}$ & $0.08 \pm 0.01$ & $\mathrm{ND}$ & $0.07 \pm 0.00$ \\
\hline 21 & Octacosanol & 3110.60 & 3157.49 & $0.37 \pm 0.00$ & $0.61 \pm 0.00$ & $\mathrm{ND}$ & $\mathrm{ND}$ & $\mathrm{ND}$ & $0.49 \pm 0.00$ \\
\hline 22 & Triacontanol & 3246.00 & 3333.67 & ND & $0.11 \pm 0.00$ & ND & ND & ND & ND \\
\hline 23 & Myristic acid & 1765.00 & 1817.65 & $0.12 \pm 0.00$ & $0.09 \pm 0.00$ & $0.18 \pm 0.00$ & $0.13 \pm 0.01$ & $\mathrm{ND}$ & $0.13 \pm 0.00$ \\
\hline 24 & Hexadecanoic acid & 1968.00 & 2065.89 & $0.24 \pm 0.01$ & $0.20 \pm 0.01$ & $0.23 \pm 0.01$ & $0.19 \pm 0.00$ & $0.03 \pm 0.00$ & $0.18 \pm 0.01$ \\
\hline 25 & Linoleic acid & 2183.00 & 2231.89 & $0.04 \pm 0.00$ & $0.03 \pm 0.00$ & ND & $0.02 \pm 0.00$ & ND & ND \\
\hline 26 & Oleic acid & 2175.00 & 2237.06 & $0.07 \pm 0.01$ & $0.05 \pm 0.00$ & $0.06 \pm 0.00$ & $0.05 \pm 0.01$ & $\mathrm{ND}$ & $0.04 \pm 0.00$ \\
\hline 27 & Stearic acid & 2167.00 & 2263.45 & $0.14 \pm 0.01$ & $0.12 \pm 0.00$ & $0.15 \pm 0.01$ & $0.10 \pm 0.00$ & ND & $0.11 \pm 0.01$ \\
\hline 28 & Arachidic acid & 2365.30 & 2462.70 & $0.08 \pm 0.01$ & $0.37 \pm 0.00$ & $0.18 \pm 0.01$ & $0.14 \pm 0.11$ & ND & $0.22 \pm 0.01$ \\
\hline 29 & Heneicosanoic acid & 2463.20 & 2562.39 & ND & $0.12 \pm 0.01$ & $0.09 \pm 0.01$ & $0.04 \pm 0.00$ & ND & $0.08 \pm 0.00$ \\
\hline 30 & Behenic acid & 2569.00 & 2667.21 & $1.58 \pm 0.02$ & $2.54 \pm 0.02$ & $2.66 \pm 0.01$ & $1.46 \pm 0.02$ & $0.41 \pm 0.02$ & $2.13 \pm 0.02$ \\
\hline 31 & Tricosanoic acid & 2668.10 & 2761.93 & $0.07 \pm 0.00$ & $0.12 \pm 0.01$ & $0.21 \pm 0.00$ & $0.07 \pm 0.00$ & ND & $0.11 \pm 0.00$ \\
\hline 32 & Tetracosanoic acid & 2763.00 & 2869.19 & $2.35 \pm 0.01$ & $2.58 \pm 0.02$ & $4.03 \pm 0.02$ & $1.74 \pm 0.02$ & $0.02 \pm 0.00$ & $2.23 \pm 0.03$ \\
\hline 33 & Pentacosanoic acid & 3345.00 & 3259.94 & $0.11 \pm 0.00$ & $0.16 \pm 0.00$ & $0.31 \pm 0.00$ & $0.08 \pm 0.01$ & ND & $0.13 \pm 0.00$ \\
\hline 34 & Hexacosanoic acid & 2962.00 & 3061.39 & $1.67 \pm 0.04$ & $1.64 \pm 0.02$ & $2.97 \pm 0.01$ & $1.22 \pm 0.01$ & $0.06 \pm 0.00$ & $1.42 \pm 0.01$ \\
\hline 35 & Hexacosanoic acid, 2-hydroxy- & 2908.00 & 2979.36 & ND & $0.03 \pm 0.00$ & ND & ND & ND & ND \\
\hline 36 & Octacosanoic acid & 3161.00 & 3241.70 & $0.46 \pm 0.01$ & $0.44 \pm 0.01$ & $0.96 \pm 0.01$ & $0.64 \pm 0.02$ & ND & $0.49 \pm 0.01$ \\
\hline 37 & 2-Mono-isobutyrin & 1324.00 & 1423.41 & $0.05 \pm 0.00$ & $0.03 \pm 0.00$ & $0.11 \pm 0.01$ & $0.06 \pm 0.00$ & $\mathrm{ND}$ & $0.02 \pm 0.00$ \\
\hline
\end{tabular}




\begin{tabular}{|c|c|c|c|c|c|c|c|c|c|}
\hline 38 & Diisobutyl phthalate & 1871.80 & 1893.41 & $0.05 \pm 0.00$ & $0.04 \pm 0.00$ & $\mathrm{ND}$ & $0.05 \pm 0.00$ & $0.01 \pm 0.00$ & $0.04 \pm 0.00$ \\
\hline 39 & Dibutyl phthalate & 1967.40 & 1986.75 & $0.04 \pm 0.00$ & $0.03 \pm 0.00$ & ND & $0.03 \pm 0.00$ & $0.05 \pm 0.01$ & $0.04 \pm 0.00$ \\
\hline 40 & 1-O-hexadecanolenin & 2563.00 & 2593.71 & $0.32 \pm 0.01$ & $0.20 \pm 0.00$ & $0.35 \pm 0.01$ & $0.17 \pm 0.01$ & $\mathrm{ND}$ & $0.18 \pm 0.01$ \\
\hline 41 & 1-Monopalmitin & 2482.00 & 2576.01 & $0.13 \pm 0.00$ & $0.07 \pm 0.01$ & $0.09 \pm 0.01$ & $0.16 \pm 0.01$ & $\mathrm{ND}$ & $0.17 \pm 0.00$ \\
\hline 42 & Glycerin octadecylate & 2681.00 & 2777.40 & $0.16 \pm 0.01$ & $0.18 \pm 0.01$ & $1.14 \pm 0.01$ & $0.09 \pm 0.00$ & $\mathrm{ND}$ & $0.17 \pm 0.01$ \\
\hline 43 & 1-Monostearin & 2681.00 & 2780.24 & $0.13 \pm 0.01$ & $0.06 \pm 0.01$ & $0.08 \pm 0.00$ & $0.12 \pm 0.00$ & $\mathrm{ND}$ & $0.12 \pm 0.01$ \\
\hline 44 & Carbonic acid, eicosyl prop-1-en-2-yl ester & 2368.00 & 2458.96 & $\mathrm{ND}$ & $\mathrm{ND}$ & $\mathrm{ND}$ & $\mathrm{ND}$ & $0.02 \pm 0.00$ & ND \\
\hline 45 & 2,3-hydroxy-propyl docosanoate & 653.00 & 727.05 & $0.08 \pm 0.00$ & $0.10 \pm 0.00$ & $0.15 \pm 0.00$ & $0.04 \pm 0.00$ & ND & $0.09 \pm 0.00$ \\
\hline 46 & 2-Hydroxy methyl pentacosanoate & 1118.00 & 1214.37 & $\mathrm{ND}$ & $\mathrm{ND}$ & $0.15 \pm 0.00$ & $\mathrm{ND}$ & $\mathrm{ND}$ & ND \\
\hline 47 & Glycerol twenty-four alkyl acid & 1757.00 & 1839.52 & ND & $0.20 \pm 0.00$ & $0.38 \pm 0.00$ & $0.08 \pm 0.00$ & ND & ND \\
\hline 48 & Kaurenoicacid & 2423.00 & 2513.02 & $0.09 \pm 0.00$ & $\mathrm{ND}$ & $\mathrm{ND}$ & $\mathrm{ND}$ & $\mathrm{ND}$ & $\mathrm{ND}$ \\
\hline 49 & 30-Norhopane & 2186.00 & 2248.50 & $0.04 \pm 0.00$ & ND & ND & ND & ND & ND \\
\hline 50 & Uvaol & 3499.00 & 3434.66 & $\mathrm{ND}$ & $\mathrm{ND}$ & $\mathrm{ND}$ & $\mathrm{ND}$ & $\mathrm{ND}$ & $0.08 \pm 0.00$ \\
\hline 51 & $\alpha$-Amyrin & 3320.00 & 3337.07 & $0.40 \pm 0.01$ & $\mathrm{ND}$ & $\mathrm{ND}$ & $\mathrm{ND}$ & $\mathrm{ND}$ & $0.17 \pm 0.00$ \\
\hline 52 & $\beta$-Lanosta-9(11),24-dien-3-ol & 3300.00 & 3348.73 & $0.04 \pm 0.00$ & $\mathrm{ND}$ & $0.09 \pm 0.00$ & ND & ND & $\mathrm{ND}$ \\
\hline 53 & Hopane & 2593.00 & 2638.61 & $0.03 \pm 0.00$ & $\mathrm{ND}$ & ND & ND & $\mathrm{ND}$ & $\mathrm{ND}$ \\
\hline 54 & 3-O-Acetyl-6-methoxy-cycloartenol & 686.00 & 765.29 & $0.11 \pm 0.00$ & ND & $0.18 \pm 0.01$ & $\mathrm{ND}$ & ND & ND \\
\hline 55 & 2,2'-Dihydroxy-4-methoxybenzophenone & 2595.00 & 2547.92 & $0.06 \pm 0.01$ & $\mathrm{ND}$ & $\mathrm{ND}$ & $\mathrm{ND}$ & $\mathrm{ND}$ & $\mathrm{ND}$ \\
\hline 56 & 2-Hexadecanal & 1798.00 & 1828.75 & ND & ND & ND & $\mathrm{ND}$ & $0.02 \pm 0.00$ & ND \\
\hline 57 & 2-Nonadecanone & 2106.00 & 2132.86 & $\mathrm{ND}$ & $0.05 \pm 0.00$ & $\mathrm{ND}$ & $0.03 \pm 0.00$ & $0.08 \pm 0.00$ & $0.10 \pm 0.00$ \\
\hline
\end{tabular}




\begin{tabular}{|c|c|c|c|c|c|c|c|c|c|}
\hline 58 & 2-Tritriacontanone & 3437.00 & 3498.68 & ND & ND & ND & ND & ND & $0.02 \pm 0.00$ \\
\hline 59 & P-Phthalic acid & 1917.00 & 1817.62 & $\mathrm{ND}$ & $\mathrm{ND}$ & $\mathrm{ND}$ & $0.03 \pm 0.00$ & $\mathrm{ND}$ & $0.03 \pm 0.00$ \\
\hline 60 & Bisphenol A monomethyl ether & 2122.00 & 2203.72 & $1.20 \pm 0.02$ & $0.65 \pm 0.01$ & $0.23 \pm 0.01$ & $0.13 \pm 0.00$ & $\mathrm{ND}$ & $0.17 \pm 0.01$ \\
\hline 61 & 3,5-di-tert-buty-4-hydroxyanisole & 1731.00 & 1785.53 & $0.04 \pm 0.00$ & $0.05 \pm 0.01$ & ND & $0.06 \pm 0.00$ & ND & $0.05 \pm 0.01$ \\
\hline 62 & 4-Hydroxybenzyl acohol & 1803.00 & 1834.71 & ND & ND & ND & ND & $0.34 \pm 0.01$ & ND \\
\hline 63 & Diethylstilbestrol & 2366.00 & 2334.62 & ND & ND & $0.14 \pm 0.00$ & $0.10 \pm 0.00$ & ND & ND \\
\hline 64 & Glycerol & 2322.00 & 2412.09 & $0.09 \pm 0.00$ & $0.08 \pm 0.00$ & $\mathrm{ND}$ & $\mathrm{ND}$ & ND & $\mathrm{ND}$ \\
\hline 65 & Phenol, 2,6-bis(1,1-dimethylethyl)- & 2327.00 & 2385.57 & $0.03 \pm 0.00$ & ND & ND & ND & ND & ND \\
\hline 66 & Anthraquinone, 2,3,6,7-tetramethyl- & 2838.00 & 2935.99 & $0.23 \pm 0.01$ & $0.61 \pm 0.01$ & $0.87 \pm 0.01$ & $0.73 \pm 0.02$ & ND & $0.74 \pm 0.01$ \\
\hline 67 & Lathosterol & 3170.00 & 3248.23 & $0.03 \pm 0.00$ & $0.04 \pm 0.00$ & $\mathrm{ND}$ & 0.00 & ND & $0.03 \pm 0.00$ \\
\hline 68 & Phenol, 2,2'-methylenebis[6-(1,1-dimethy lethyl)-4-methyl- & 2398.00 & 2440.09 & ND & ND & ND & ND & $0.07 \pm 0.00$ & $0.04 \pm 0.00$ \\
\hline 69 & [(3,3-dimethyl-3H,7H-furo[3,2-c:5,4-f']bis[1]benzopyran-6b,10(12bH)-di & 1532.00 & 1609.73 & $0.03 \pm 0.00$ & $0.07 \pm 0.00$ & ND & $0.06 \pm 0.00$ & ND & $0.07 \pm 0.00$ \\
\hline 70 & 7,9-Di-tert-butyl-1-oxaspiro(4,5)deca-6,9-diene-2,8-dione & 1916.00 & 2011.09 & $0.07 \pm 0.00$ & $0.04 \pm 0.00$ & $\mathrm{ND}$ & $0.06 \pm 0.00$ & $0.07 \pm 0.00$ & $0.08 \pm 0.00$ \\
\hline
\end{tabular}

Note: The data in Table S2 represents the wax content $\left(\mu \mathrm{g} / \mathrm{cm}^{2}\right)$ of goji berries, all of which are average \pm standard deviation (SD). $\mathrm{RI}^{*}$ is the retrieved value of NIST17, and $\mathrm{RI}^{* *}$ is the measured value. ND, not detected. 
Table S3. Components and concentrations of cuticular wax in different species of Lycium barbarum $\mathrm{L}$.

\begin{tabular}{|c|c|c|c|c|c|c|c|c|}
\hline species & Alkanes $\left(\mu \mathrm{g} / \mathrm{cm}^{2}\right)$ & Primary alcohols $\left(\mu \mathrm{g} / \mathbf{c m}^{2}\right)$ & Fatty acids $\left(\boldsymbol{\mu g} / \mathbf{c m}^{2}\right)$ & Esters $\left(\mu \mathrm{g} / \mathbf{c m}^{2}\right)$ & Terpenoids $\left(\mu \mathrm{g} / \mathbf{c m}^{2}\right)$ & Ketones $\left(\mu \mathrm{g} / \mathbf{c m}^{2}\right)$ & Others $\left(\mu \mathrm{g} / \mathrm{cm}^{2}\right)$ & Total wax $\left(\mu \mathrm{g} / \mathbf{c m}^{2}\right)$ \\
\hline Ningqi-1 & $3.15 \pm 0.04 \mathrm{a}$ & $1.60 \pm 0.03 \mathrm{~d}$ & $6.92 \pm 0.10 \mathrm{~d}$ & $0.96 \pm 0.02 \mathrm{~b}$ & $0.71 \pm 0.01 \mathrm{a}$ & $0.06 \pm 0.01 \mathrm{c}$ & $1.72 \pm 0.02 \mathrm{a}$ & $15.11 \pm 0.17 \mathrm{c}$ \\
\hline Ningqi-5 & $2.06 \pm 0.03 \mathrm{c}$ & $2.29 \pm 0.01 \mathrm{~b}$ & $8.49 \pm 0.02 \mathrm{~b}$ & $0.92 \pm 0.01 \mathrm{~b}$ & $0.00 \pm 0.00 \mathrm{~d}$ & $0.05 \pm 0.00 \mathrm{~d}$ & $1.54 \pm 0.01 \mathrm{~b}$ & $15.48 \pm 0.06 \mathrm{~b}$ \\
\hline Ningnongqi-9 & $2.02 \pm 0.04 \mathrm{c}$ & $2.99 \pm 0.02 \mathrm{a}$ & $12.02 \pm 0.08 \mathrm{a}$ & $2.45 \pm 0.04 \mathrm{a}$ & $0.26 \pm 0.01 \mathrm{~b}$ & $0.00 \pm 0.00 \mathrm{f}$ & $1.24 \pm 0.00 \mathrm{c}$ & $20.98 \pm 0.19 \mathrm{a}$ \\
\hline Z168 & $1.01 \pm 0.03 \mathrm{~d}$ & $1.37 \pm 0.02 \mathrm{e}$ & $5.87 \pm 0.05 \mathrm{e}$ & $0.80 \pm 0.03 \mathrm{c}$ & $0.00 \pm 0.00 \mathrm{~d}$ & $0.03 \pm 0.00 \mathrm{e}$ & $1.20 \pm 0.02 \mathrm{~d}$ & $10.28 \pm 0.15 \mathrm{e}$ \\
\hline Z44 & $2.02 \pm 0.02 \mathrm{c}$ & $0.10 \pm 0.01 \mathrm{f}$ & $0.53 \pm 0.02 \mathrm{f}$ & $0.08 \pm 0.01 \mathrm{~d}$ & $0.00 \pm 0.00 \mathrm{~d}$ & $0.11 \pm 0.00 \mathrm{~b}$ & $0.48 \pm 0.01 \mathrm{e}$ & $3.32 \pm 0.02 \mathrm{f}$ \\
\hline $16-23-7-8$ & $2.17 \pm 0.02 \mathrm{~b}$ & $2.24 \pm 0.00 \mathrm{c}$ & $7.27 \pm 0.01 \mathrm{c}$ & $0.83 \pm 0.01 \mathrm{c}$ & $0.25 \pm 0.01 \mathrm{c}$ & $0.12 \pm 0.00 \mathrm{a}$ & $1.21 \pm 0.03 \mathrm{~d}$ & $14.09 \pm 0.02 \mathrm{~d}$ \\
\hline Total & $2.07 \pm 0.68$ & $1.77 \pm 1.00$ & $6.85 \pm 3.76$ & $1.01 \pm 0.78$ & $0.20 \pm 0.28$ & $0.06 \pm 0.05$ & $1.23 \pm 0.42$ & $13.21 \pm 5.94$ \\
\hline
\end{tabular}

Note: Different lowercase letters of the same factor in the same column indicate significant differences $(P<0.05)$. 